\title{
Prioritization of looming and receding objects: Equal slopes, different intercepts
}

\author{
Paul A. Skarratt \\ Durham University, Durham, England \\ and University of Hull, Hull, England \\ Geoff G. Cole \\ Durham University, Durham, England \\ AND \\ Angus R. H. Gellatly \\ Oxford Brookes University, Oxford, England
}

\begin{abstract}
Franconeri and Simons (2003) reported that simulated looming objects (marked by a size increase) captured attention, whereas simulated receding objects (marked by a size decrease) did not. This finding has been challenged with the demonstration that receding objects can capture attention when they move in three-dimensional depth. In the present study, we compared the effects of objects that either loomed or receded in depth. The results of two experiments showed that whereas both motion types benefited from attentional prioritization, as judged by their search slopes, looming objects elicited shorter response times (RTs). We conclude that both motion types attract attention during search; however, the RT advantage for looming motion seems to reflect a processing enhancement that occurs outside of selection and is conferred on the basis of motion direction.
\end{abstract}

Attention is often said to be the ability to select certain aspects of the environment in preference to others. Since we are unable to attend to all stimulus information at once, we select only what is likely to be behaviorally important. Although the selection of these stimuli is largely determined by a person's intentions and goals (see, e.g., Folk, Remington, \& Johnston, 1992; Folk, Remington, \& Wright, 1994), it has long been argued that certain stimulus features, such as the abrupt onset of a stimulus object, have the potential to summon attention automatically (e.g., Yantis \& Jonides, 1984). More recently, there has been considerable debate regarding the types of motion information that might also attract attention automatically. In a series of exchanges, Franconeri and Simons $(2003,2005)$ and Abrams and Christ (2003, 2005a, 2005b, 2006) have emphasized the relative importance, respectively, of motion per se and of motion onset. That is, is attention as likely to be attracted to a moving object during the object's motion as it is to be attracted upon commencement of the motion? Further embedded in this debate is the question of whether motion direction can be influential in the marshalling of attention. That is, does looming motion summon attention more effectively than does receding motion? This question is the focus of interest in the present study.

The notion that the human visual system might be preferentially sensitive to looming motion is persuasive. Studies of animal neurophysiology have revealed neurons in the visual cortex of rhesus monkeys that selectively respond to stimuli that simulate motion toward or away from the animal (Zeki, 1974a, 1974b). Moreover, single-cell recordings in the medial superior temporal area of the macaque monkey have also revealed cells that are sensitive to motion direction, many more of which respond to looming than to receding motion (Tanaka \& Saito, 1989). These findings concur with those from animal behavior studies that have shown that the rapid expansion (i.e., looming) of circular shadows elicits fear responses in adult and infant rhesus monkeys, whereas rapidly contracting (receding) shadows do not (Schiff, Caviness, \& Gibson, 1962). Human infants, too, show sensitivity to looming motion. Schmuckler, Collimore, and Dannemiller (2007) recently compared the blink responses of 4- to 5-month-old infants who perceived objects looming on collision or near-miss trajectories. The results showed that objects on a collision course elicited a greater number of blink responses than did those on noncollision trajectories, suggesting that infants are able to discriminate subtle differences in motion direction. Additionally, Jouen (1990; see also Jouen, Lepecq, Gapenne, \& Bertenthal, 2000) showed that even 3 -day-old neonates responded to looming flow motion patterns by tilting their heads backward. Interestingly, the extent to which the neonates tilted their heads correlated positively with the optic flow velocity of the looming stimuli. Taken together, these results, in light of the

P. A. Skarratt, p.skarratt@hull.ac.uk 
perceptual inexperience of the infants tested, suggest that sensitivity to looming motion might be hardwired.

However, the evidence that looming motion can be influential in the distribution of attention is mixed. For example, Franconeri and Simons (2003) reported an experiment in which objects that appeared to loom toward the observer attracted attention, whereas receding objects did not, suggesting that motion direction is important in marshalling attention. Their study employed a search task commonly used in studies of attentional priority. In such tasks, participants are asked to search arrays for a specific target that occurs among distractors, when one randomly selected item is a unique singleton that coincides with the target as often as it does with the distractors (e.g., Egeth \& Yantis, 1997). Thus, participants do not accrue an advantage by deliberately searching for the unique item first. However, Franconeri and Simons's (2003) results showed that identification of the target was facilitated whenever it coincided with the looming stimulus. This important finding therefore suggests that attention is attracted to looming objects automatically.

However, some questions remain over the effectiveness of the method Franconeri and Simons (2003) used to simulate looming and receding motion, which involved the expansion (looming) or contraction (receding) of twodimensional objects. Abrams and Christ (2005b) pointed out that size change provides only one cue to depth, leaving open the possibility that participants had interpreted the stimuli as "expanding" or "shrinking" rather than "looming" or "receding." To provide a stronger test of whether receding objects can attract attention, they generated receding singletons stereoscopically, combining size changes with binocular disparity to create apparent motion in depth. Under these conditions, receding targets did attract attention, suggesting that moving stimuli are prioritized on the basis of their motion onset rather than that of the direction in which they move. This result challenges the generality of the previous finding, but only by posing a further puzzle. As Franconeri and Simons (2005) argued, if motion onset is the critical stimulus for attentional capture, the question remains as to why expanding but not contracting stimuli were able to attract attention in their experiment, even though both types of stimuli generated a motion onset. At the very least, the Franconeri and Simons (2003) data suggest that the visual system may in some way be differentially sensitive to looming versus receding motion.

A study by von Mühlenen and Lleras (2007) also supports the notion that looming motion receives attentional priority. Their experiments utilized arrays composed of randomly moving dot patterns. At some point in each trial, the dot motion in one half of the array gradually transformed from random into looming or receding patterns, while dots in the other half of the array maintained their random motion. Looming motion was simulated by the dots emanating outward from a vanishing point positioned centrally in that half of the array, whereas receding motion was rendered by dots retracting inward. An attentional preference for either motion type was probed on each trial with a target appearing at the center of one of the two motion arrays. The results showed that relative to detection in the random-motion arrays, performance was facilitated for targets in the looming but not the receding arrays.

The present study set out to examine further the central issue of what sort of motion in depth attracts attention. The study was motivated by the current disagreement regarding whether motion direction is influential in guiding attention. The studies cited above used a variety of methods to simulate looming and receding motion, such as the expansion/contraction of two-dimensional figures (Franconeri \& Simons, 2003), random dot patterns (von Mühlenen \& Lleras, 2007), and binocular disparity to generate stereoscopic depth (Abrams \& Christ, 2005b). However, because Abrams and Christ (2005b) set out only to test whether receding objects are capable of attracting attention, they did not include looming objects in their experiment. Therefore, it is not clear how attention is deployed in stereoscopic displays that contain both looming and receding objects. The demonstration that objects stereoscopically presented as receding in depth can attract attention is nonetheless important, because it shows that receding objects can be prioritized ahead of static objects. However, a direct comparison of looming and receding motion in stereo depth is required in order to establish similarities and differences between the two with respect to the attentional priority they receive. Hence, in the present study we examined the effects of looming and receding objects in two variations of Abrams and Christ's (2005b) experiment. We show that both motion types attract attention during search, but that looming objects benefit from more rapid processing overall.

\section{EXPERIMENT 1}

Our first experiment was a modification of Abrams and Christ's (2005b) Experiment 1, which assessed the ability of receding motion to capture attention. In their experiment, participants viewed through a stereoscope arrays containing three or six figure-eight placeholder shapes. This allowed one of the placeholders to be manipulated so it appeared to "float" in front of the others. Receding motion was simulated by having this placeholder move back into alignment with the other figures (see Figure 1). All of the placeholders then transformed into letters by shedding two of their segments, and participants searched for a target that appeared with the distractors. The present experiment was based on theirs, but with trials containing either a looming or a receding placeholder. Looming-object trials followed the same basic procedure as receding-object trials, except that the looming figure appeared to float behind the other placeholders at the beginning of the trial. This figure then moved forward prior to transformation into the search array.

\section{Method}

Participants. Fifteen undergraduates participated in the experiment. All had normal or corrected-to-normal binocular vision and were naive to the purposes of the experiment. Each received $£ 5$ for their participation.

Apparatus and Stimuli. The experiment was conducted on a Pentium PC linked to a $60-\mathrm{Hz}$ color monitor. Participants viewed the display through a stereoscope that restricted the view of each eye to only half of the display, thus allowing binocular disparity to be used 


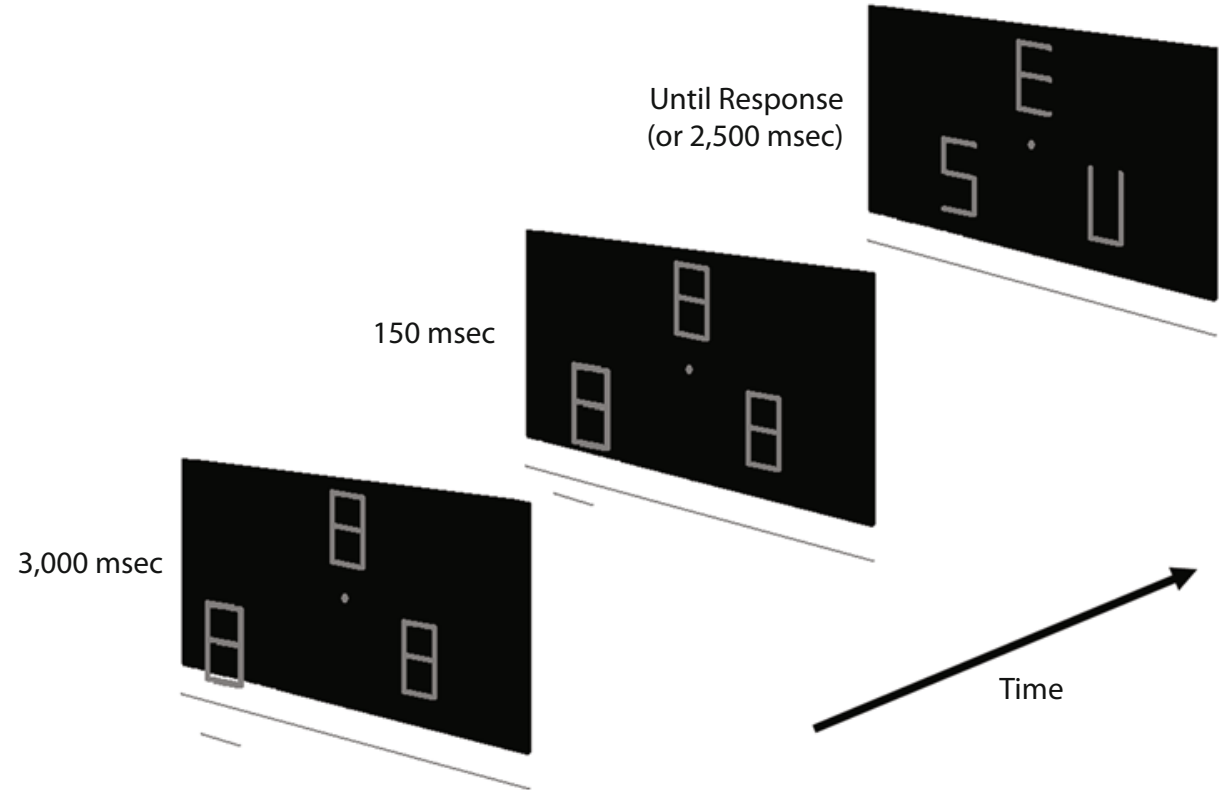

Figure 1. Sequence of events on a trial during Abrams and Christ's (2005b), as well as the present, Experiment 1. The figure depicts a receding placeholder, which began the trial "in front" of the others. The placeholder then receded over a period of $150 \mathrm{msec}$, after which all the placeholders were transformed into letters. See the main text for additional information.

to simulate depth. The trial sequence is shown in Figure 1. Each trial began with a fixation display composed of either three or six figureeight placeholders positioned at the vertices of an imaginary triangle or hexagon (with one placeholder always occupying the 12 o'clock position). When viewed from $57 \mathrm{~cm}$, the stimulus measurements were identical to those used by Abrams and Christ (2005b). All items in the display were presented at zero disparity, with the exception of one randomly selected placeholder, which appeared either in front of or behind the other placeholders. The retinal size of this placeholder was adjusted to maintain size constancy, as well as size consistency, with the other placeholders. In other words, looming and receding objects changed retinal size (on the monitor screen) in accordance with their apparent depth. After a fixation array lasting 3,000 msec, the motion placeholder moved in depth until zero disparity was reached and it became aligned with the other placeholders. This motion was completed in $150 \mathrm{msec}$, after which all placeholders transformed into letters by each shedding two or three of their segments. One of the placeholders became a target letter (either an "S" or an "H"), whereas the others became distractors (randomly selected from "E," "U," "P," "C," or "F"). Participants identified the target by pressing one of two keys on a standard keyboard as quickly and as accurately as possible. The response key mapping was reversed for half of the participants. The search array remained until a response was made or 2,500 msec had elapsed. Participants were instructed to maintain fixation throughout each trial.

Design and Procedure. A $2 \times 3$ fully related design was employed, with array size (three or six) and target motion type (looming, receding, or static placeholder) as the two factors. Participants undertook a single block of 576 trials. The trials were divided equally into array sizes of three and six. Half of the trials for each size contained a looming, and the other half a receding, placeholder. The target was equally likely to be an "S" or an " $\mathrm{H}$ " and was transformed from the looming figure on one third of all array-size-three trials and on one sixth of array-size-six trials. The same was true for the receding figure. Thus, the target was equally likely to be associated with any figure in the display, whether looming, receding, or stationary, ${ }^{1}$ and participants were made aware of this. Distractors could be any combination of nontarget letters for each target letter. Before testing, participants were given a single block of 24 practice trials, and they were offered a break after every 96 trials in the experiment proper.

\section{Results and Discussion}

Outliers were excluded from the condition means. An outlier was defined as a response time (RT) more than two standard deviations above or below the condition mean for each participant. This exclusion resulted in the removal of $4.6 \%$ of all responses from the analysis.

Mean RTs are shown in Figure 2. As can be seen, RT increased considerably as a function of array size in the static condition (search slope of $34 \mathrm{msec} / \mathrm{item}$ ). By contrast, the search slopes for looming and receding targets were 15.7 and $15.3 \mathrm{msec} / \mathrm{item}$, respectively. These slopes show that both motion types received equal attentional priority relative to static targets. However, overall RTs were shortest for looming targets. These data were submitted to a $2 \times 3$ within-participants ANOVA with array size (three or six) and motion type (looming, receding, or static) as the two factors. We obtained significant main effects of array size $[F(1,14)=45.9, p<.001]$ and motion type $[F(2,28)=37.1, p<.001]$ and a significant interaction $[F(2,28)=6.7, p<.05]$. Accuracy was above $94 \%$ in all conditions and did not differ according to condition (all $p \mathrm{~s}>.05$ ).

Planned comparisons focused on the RT differences between looming and receding targets as a function of array size. We therefore reanalyzed the data using a $2 \times 2$ ANOVA, omitting the static condition. Again, the effect of set size was significant $[F(1,14)=23.8, p<.001]$. The interaction was not significant $[F(1,14)<1]$, but responses to looming targets were faster than those to receding targets $[F(1,14)=7.4, p<.02]$. 


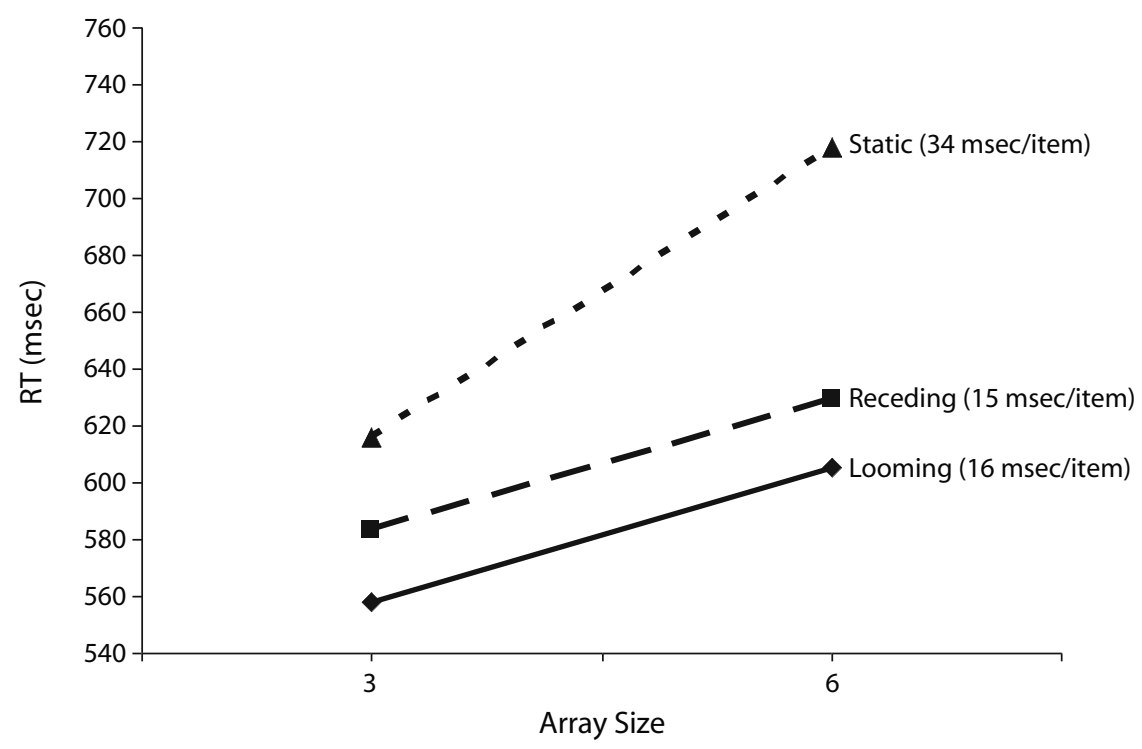

Figure 2. Mean response times (RTs) to looming, receding, and static targets as a function of array size in Experiment 1. Slopes are labeled according to target type, and the slope values are given in parentheses.

The results of Experiment 1 are twofold. The similarity in search slopes for looming and receding targets indicates equivalence in their ability to attract attention. However, RTs were shortest for looming targets, indicating a processing advantage over receding targets.

Two possible explanations for the RT advantage of looming targets suggest themselves immediately. One possibility is that looming objects receive enhanced processing before or after initial selection has taken place (see, e.g., Harris, Shaw, \& Bates, 1979; Wolfe, 2003; Wolfe, Oliva, Horowitz, Butcher, \& Bompas, 2002). That is, the RT advantage may lie not in the ability of looming objects to capture attention, but in the processing they receive pre- or postselection. Alternatively, looming motion may elicit a general alerting effect that primes motor responses (e.g., Hackley \& Valle-Inclán, 2003). Fernandez-Duque and Posner (1997) reported a similar effect that has been shown to modulate visual search performance. In their experiment, targets were preceded on some trials by a cue that occurred simultaneously in the top, bottom, left, and right quadrants of the array. Hence, the cue offered no taskrelevant information as to the location of the target, and as such did not encourage orienting to any specific location in the array. However, the results showed that target responses were generally faster on cue trials than on noncue trials, but that RTs did not differ according to the spatial proximity between any of the cue positions and the target. In other words, responses on cue trials were similarly rapid, irrespective of the actual location of the target. This effect is typical of a general alerting effect that is spatially broad and nonattentional in nature (cf. Posner, 1980).

It is thus possible that the results of Experiment 1 could be explained by a general alerting effect elicited by looming objects. If so, the increase in arousal would have speeded RTs on those trials only, leading to the RT advantage for those targets. The net result would therefore have been a main RT effect of target motion type, but without a difference in search slopes. However, an alerting effect of looming motion should also have speeded RTs to static targets appearing on looming trials, relative to those appearing on receding trials. This would not be apparent from Figure 2, since RTs to static targets on looming and receding trials were combined to provide those data. In a further analysis, therefore, RTs to static targets were compared as a function of their presence on looming or receding trials. No difference was observed $(F<1$; for static targets, $M=664$, $S D=115$, on looming trials vs. $M=669, S D=115$, on receding trials). This null effect appears to refute an alerting explanation for the looming advantage found in Experiment 1 . However, the possibility was tested empirically in Experiment 2 by having looming and receding objects compete for attention within the same trials.

\section{EXPERIMENT 2}

The search slopes from Experiment 1 revealed that looming and receding targets received equal prioritization ahead of static targets, but that looming targets elicited faster overall RTs. These data were obtained with the two motion types occurring in separate trials. A more direct examination of the propensities of looming and receding motion to summon attention would be to present them in competition against each other within the same trials.

In Experiment 2, looming, receding, and static objects occurred within the same arrays. This was a conceptual replication of Abrams and Christ (2003; see also Abrams \& Christ, 2005b), in which several different motion transients were set in direct competition for attentional priority. This manipulation allowed us to examine the alerting effect theorized as possibly accounting for the looming advan- 
tage in Experiment 1: If a looming stimulus does speed RTs via an alerting effect, it should also speed responses to the receding and static targets, leading to the abolition of the looming advantage. Thus, Experiment 2 was identical to Experiment 1, with the exception that every trial contained a looming and a receding placeholder within the array. The remaining elements were made up of static placeholders.

\section{Method}

Participants. Eighteen undergraduates who had not served previously participated in the experiment.

Apparatus and Stimuli. This experiment was identical to Experiment 1 , with the exception that the looming, receding, and static elements appeared within the same trials.

Design and Procedure. Participants undertook a single block of 288 trials, with breaks offered every 96 trials. All placeholders in the array were equally likely to become a target, irrespective of whether they loomed, receded, or remained static.

\section{Results and Discussion}

Outliers were excluded as in Experiment 1. This resulted in the removal of $5.1 \%$ of all responses. The mean RTs are shown in Figure 3. As can be seen, the RT data are almost identical to those obtained in Experiment 1, showing a steeper search slope for static targets $(43 \mathrm{msec} / \mathrm{item})$ and shallower slopes for looming and receding targets $(22.7$ and $25.7 \mathrm{msec} /$ item, respectively). These observations were corroborated using a $2 \times 3$ within-subjects ANOVA, with array size (three or six) and motion type (looming, receding, or static) as the two factors. We obtained significant main effects of array size $[F(1,17)=48.3, p<.001]$ and motion type $[F(2,34)=36.7, p<.001]$. The interaction was also significant $[F(2,34)=10.7, p<.001]$. Accuracy was above $95 \%$ in all conditions and was analyzed in the same way as the RT data. Only the effect of motion type reached significance $[F(2,34)=8.7, p=.001]$. Accuracy was similar for looming and receding targets (98\% and $97 \%$, respectively) $[t(17)=1.5, p>.05]$. However, a comparison of receding versus static targets revealed poorer accuracy for static targets $(95 \%)[t(17)=2.5, p<.03]$. There was, therefore, no evidence of any speed-accuracy trade-off.

As in Experiment 1, planned comparisons focused on looming and receding motion across array sizes. The corresponding RT data were reanalyzed using a $2 \times 2$ ANOVA, with the static target conditions omitted. Again, significant main effects were obtained for array size $[F(1,17)=30.4$, $p<.001]$ and motion type $[F(1,17)=21.98, p<.001]$. However, the interaction was not significant $[F(1,17)<1]$. The findings of Experiment 2, in which looming and receding objects were pitted against each other, proved entirely consistent with those of Experiment 1, in which the two motion types were presented in competition only with static items. Looming and receding targets again attracted attention relative to static targets, but looming targets were identified more rapidly. These results show that the generalalerting hypothesis cannot account for the RT advantage for looming motion, since an alerting effect would have speeded RTs to the receding and static targets also. However, there was again no difference in the search slopes for looming and receding motion, indicating that the processing advantage enjoyed by looming motion cannot be due to a straightforward primacy during selection. In the following section, we elaborate on possible explanations for the looming advantage and consider the impact of our findings on the debate over when and how moving stimuli attract attention.

\section{GENERAL DISCUSSION}

The present experiments sought to reexamine the claim that objects that appear to loom toward the observer attract attention, whereas receding objects do not (Franconeri

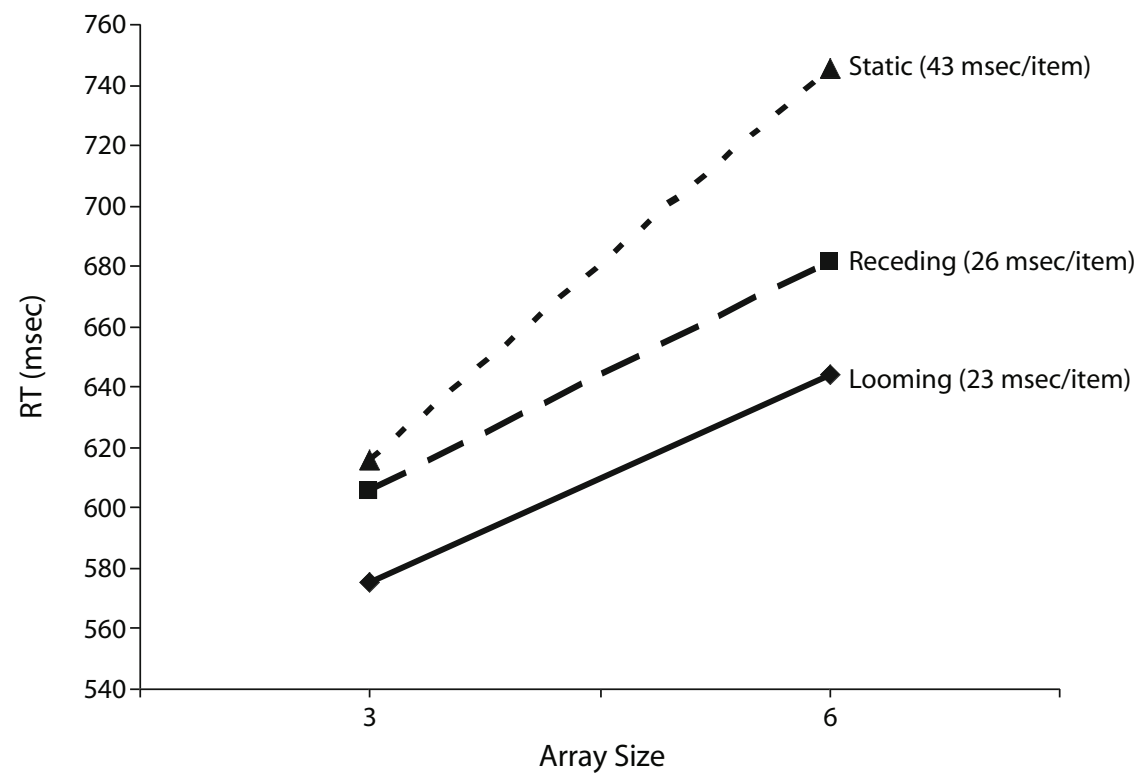

Figure 3. Mean response times (RTs) to looming, receding, and static objects as a function of array size in Experiment 2. Slopes are labeled according to target type, and the slope values are given in parentheses. 
\& Simons, 2003). This claim has been challenged by the demonstration that receding objects can attract attention when their motion is rendered in three-dimensional depth (Abrams \& Christ, 2005b). Our Experiment 1 confirmed Abrams and Christ's (2005b) finding that receding objects are capable of summoning attention when in competition with static objects, as demonstrated by a shallower search slope for receding than for static targets. In addition, however, although looming and receding targets exhibited equal search slopes, we observed faster RTs to the former than to the latter. Experiment 2 further examined this feature of Experiment 1's results. Once again, the search slopes for looming and receding motion did not differ, but an RT advantage for looming motion was manifest as a main effect. This observation seems to preclude the explanation that looming motion simply captures attention more efficiently than does receding motion, since nondiverging search slopes suggest equivalence in their selectional priority. We therefore speculated that the looming advantage might instead be caused by a general alerting effect. We tested this possibility by presenting looming, receding, and static objects within the same arrays. Any alerting effect caused by looming motion should also have speeded RTs to receding and static targets, thus abolishing the looming advantage. However, the results of Experiment 2 were similar to those of Experiment 1. Again, both motion types were prioritized ahead of static objects, but looming targets produced shorter RTs. These results clearly show that a general alerting effect cannot account for the looming advantage.

It is interesting that the effect of looming motion manifests as a speeding of RTs rather than as a shallower search function. This RT difference could be taken as an indicator that attention is captured more efficiently by looming than by receding targets, but this argument quickly runs into difficulty. By long tradition, efficiency of capture is assessed by the search function that a stimulus yields; a flatter slope indicates that the stimulus has received greater attentional priority (Simons, 2000; Treisman, 1986; Wolfe, 1998; Yantis \& Jonides, 1984). In both of the present experiments, a large set-size effect occurred, as well as an interaction with target type, which is generally taken to signal the attentional priority of moving items relative to static items. If a search slope difference is used to deduce the attentional priority of moving over static stimuli, the absence of such a difference can hardly be ignored when comparing the two types of motion. It would be inconsistent to use one criterion for identifying attentional priority when comparing moving and static targets, and another when comparing one type of moving target against another. Of course, if we adopt the slope difference criterion for all comparisons, there remains the problem of explaining the difference in absolute RTs. A convincing explanation for the RT difference is not easy to find, but the possibilities seem better than they would be for explaining why, within the same experiments, attentional priority should sometimes be manifested as a slope difference and sometimes only as an intercept difference. Whereas slope differences are not easily explained except in terms of prioritized selection, intercept differences can have various causes (Sternberg, 1969). If we accept that the similarity of slopes for looming and receding motion in both experiments indicates that the two motion types are treated equally during selection, the enhancement of processing elicited by looming objects must occur at some stage other than selection.

At this juncture, it is worth acknowledging that the looming/receding factor in our experiments can be said to have been confounded with another stimulus feature: ${ }^{2}$ Looming and receding items necessarily appeared initially in different depth planes from the static items. Therefore, they were depth as well as motion singletons in Experiment 1 , and in the array-size-six condition of Experiment 2 (when they always appeared together in the same trial), the two stood on either side of the default depth plane of the other four items. Franconeri and Simons (2005) suggested that observers may direct attention toward a stereo-depth singleton and could then track it attentively as it moves. From this suggestion, it follows that in our experiments and those of Abrams and Christ (2005b), depth location differences may have been attentionally prioritized rather than motions in depth. Experimentally separating these factors would be far from simple, since any comparison of static items with items moving in depth must always be associated with initial or final differences in depth location. It is also worth noting that this account requires that objects closer than the default plane would have equal priority in terms of slope functions but that objects farther away would be associated with the advantage in absolute RTs. This notion is contrary to the findings of O'Toole and Walker (1997), the only study of which we are aware that bears directly on the issue. Although O'Toole and Walker did not examine the pop-out effects of singletons appearing in near versus far depth planes per se, inspection of their data indicates that depth singletons were more rapidly detected when they appeared in front of their accompanying distractors than when they appeared behind. Therefore, it seems unlikely that the initial depth position of the looming and receding items, rather than their subsequent motion, could be responsible for our findings.

The conclusion suggested above, that looming objects may enjoy a processing advantage outside of attentional selection, is consistent with certain views of how serial and parallel mechanisms might interact during visual search. For example, Wolfe et al. (2002; see also Harris et al., 1979) proposed that search items might be processed in parallel, even if they are selected by attention in series. Wolfe (2003) likened this process to a car wash that is large enough to accommodate several cars at once but that can only accept the cars in series. Once inside, the speed at which a car passes through the system would depend on the efficiency of the wash, rather than the order in which it entered the car wash. Thus, it is possible that a car might exit first, even though it arrived in the system relatively late, or vice versa. In the same way, looming objects might pass through the system more efficiently than do receding objects, even though there is no difference in the way the two motion types are prioritized for selection. In other words, directional information may influence processing on either side of selection. If this is indeed the case, the results of the present experiments can serve a useful purpose in constraining future models of visual search. In demonstrating 
a novel and replicable pattern of search data, they provide a challenge for future models to accommodate.

Why might looming motion be associated with more rapid processing at some stage in the search process? Franconeri and Simons (2003) proposed a speculative hypothesis to explain why, in their experiment, looming motion captured attention but receding motion did not. According to their behavioral urgency hypothesis, certain dynamic events - such as those involving the appearance of a new object, the sudden movement of an existing object, or an object that looms toward the observer - are usually indicative of an emerging threat in the visual environment, and as such elicit an urgent attentional response. Receding objects, on the other hand, do not represent a threat, and consequently do not capture attention. Franconeri and Simons (2005) conceded that this speculative account cannot accommodate the prioritization of receding targets. However, enhanced processing of looming relative to receding objects, even without selectional advantage, could occur in the context of self-locomotion and the avoidance of collision; one can collide with a looming, but not with a receding object, and rapid action may sometimes be called for. This additional rationale for the urgency hypothesis strengthens the case for why looming motion might be associated with some sort of enhanced processing, and it may account for the specialization of neocortical cells that respond to such motion (Tanaka \& Saito, 1989; Zeki, 1974a, 1974b). More immediately, however, our results indicate that this enhancement is not implemented as prioritized selection.

\section{AUTHOR NOTE}

This work was supported by an ESRC Grant RES-000-22-1766 awarded to G.G.C. The authors thank Jeremy Wolfe, Tom Carr, Diego Fernandez-Duque, Dan Simons, and an anonymous reviewer for their helpful comments on an earlier version of the manuscript. Correspondence related to this article may be sent to P. A. Skarratt, Department of Psychology, Applied Sciences 3, University of Hull, Cottingham Road, Hull HU6 7RX, England (e-mail: p.skarratt@hull.ac.uk).

\section{REFERENCES}

Abrams, R. A., \& Christ, S. E. (2003). Motion onset captures attention. Psychological Science, 14, 427-432.

Abrams, R. A., \& Christ, S. E. (2005a). Onset but not offset of irrelevant motion disrupts inhibition of return. Perception \& Psychophysics, 67, 1460-1467.

Abrams, R. A., \& Christ, S. E. (2005b). The onset of receding motion captures attention: Comment on Franconeri and Simons (2003). Perception \& Psychophysics, 67, 219-223.

Abrams, R. A., \& Christ, S. E. (2006). Motion onset captures attention: A rejoinder to Franconeri and Simons (2005). Perception \& Psychophysics, 68, 114-117.

EgETh, H. E., \& YANTIS, S. (1997). Visual attention: Control, representation, and time course. Annual Review of Psychology, 48, 269-297.

Fernandez-Duque, D., \& Posner, M. I. (1997). Relating the mechanisms of orienting and alerting. Neuropsychologia, 35, 477-486.

Folk, C. L., Remington, R. W., \& Johnston, J. C. (1992). Involuntary covert orienting is contingent on attentional control settings. Journal of Experimental Psychology: Human Perception \& Performance, 18, 1030-1044.

Folk, C. L., Remington, R. W., \& Wright, J. H. (1994). The structure of attentional control: Contingent attentional capture by apparent motion, abrupt onset, and color. Journal of Experimental Psychology: Human Perception \& Performance, 20, 317-329.
Franconeri, S. L., \& Simons, D. J. (2003). Moving and looming stimuli capture attention. Perception \& Psychophysics, 65, 999-1010.

Franconeri, S. L., \& Simons, D. J. (2005). The dynamic events that capture visual attention: A reply to Abrams and Christ (2005). Perception \& Psychophysics, 67, 962-966.

HACKLEY, S. A., \& VALLE-InCLÁN, F. (2003). Which stages of processing are speeded by a warning signal? Biological Psychology, 64, 27-45.

Harris, J. R., ShaW, M. L., \& BATES, M. (1979). Visual search in multicharacter arrays with and without gaps. Perception \& Psychophysics, 26, 69-84.

Jouen, F. (1990). Early visual-vestibular interactions and postural development. In H. Bloch \& B. I. Bertenthal (Eds.), Sensory-motor organizations and development in infancy and early childhood (pp. 199215). Dordrecht: Kluwer.

Jouen, F., LepecQ, J.-C., Gapenne, O., \& Bertenthal, B. I. (2000). Optic flow sensitivity in neonates. Infant Behavior \& Development, 23, 271-284

O'ToOle, A. J., \& WALKer, C. L. (1997). On the preattentive accessibility of stereoscopic disparity: Evidence from visual search. Perception \& Psychophysics, 59, 202-218.

Posner, M. I. (1980). Orienting of attention. Quarterly Journal of Experimental Psychology, 32, 3-25.

Schiff, W., Caviness, J. A., \& Gibson, J. J. (1962). Persistent fear responses in rhesus monkeys to the optical stimulus of "looming." Science, 136, 982-983.

Schmuckler, M. A., Collimore, L. M., \& DANnemiller, J. L. (2007). Infants' reactions to object collision on hit and miss trajectories. Infancy, 12, 105-118.

Simons, D. J. (2000). Attentional capture and inattentional blindness. Trends in Cognitive Sciences, 4, 147-155.

STERnBerg, S. (1969). Memory-scanning: Mental processes revealed by reaction-time experiments. American Scientist, 57, 421-457.

TANaKa, K., \& SaIto, H. (1989). Analysis of motion of the visual field by direction, expansion/contraction, and rotation cells clustered in the dorsal part of the medial superior temporal area of the macaque monkey. Journal of Neurophysiology, 62, 626-641.

Treisman, A. (1986). Features and objects in visual processing. Scientific American, 255, 114B-125.

VON MÜhlenen, A., \& Lleras, A. (2007). No-onset looming motion guides spatial attention. Journal of Experimental Psychology: Human Perception \& Performance, 33, 1297-1310.

Wolfe, J. M. (1998). Visual search. In H. Pashler (Ed.), Attention (pp. 13-73). Hove, U.K.: Psychology Press.

Wolfe, J. M. (2003). Moving towards solutions to some enduring controversies in visual search. Trends in Cognitive Sciences, 7, 70-76.

Wolfe, J. M., Oliva, A., Horowitz, T. S., Butcher, S. J., \& BomPAS, A. (2002). Segmentation of objects from backgrounds in visual search tasks. Vision Research, 42, 2985-3004.

YANTIS, S., \& Jonides, J. (1984). Abrupt visual onsets and selective attention: Evidence from visual search. Journal of Experimental Psychology: Human Perception \& Performance, 10, 601-621.

ZeKI, S. M. (1974a). Cells responding to changing image size and disparity in the cortex of the rhesus monkey. Journal of Physiology, 242, 827-841.

ZEKI, S. M. (1974b). Functional organization of a visual area in the posterior bank of the superior temporal sulcus of the rhesus monkey. Journal of Physiology, 236, 549-573.

\section{NOTES}

1. Note that motion had been completed prior to the transformation of placeholders into targets and distractors. Thus, targets and distractors were associated with the motion type rather than undergoing motion themselves. However, we use the terms looming targets and receding targets hereafter for convenience.

2. We are grateful to reviewers Dan Simons and Diego FernandezDuque for drawing this confound to our attention.

(Manuscript received April 1, 2007; revision accepted for publication December 30, 2008.) 